\title{
Research Paper: Crocin Treatment after Maternal Hypoxia Attenuates Spatial Memory Impairment and Expression of BACE1 and HIF-1 $\alpha$ in Rat Offspring Brain
}

\author{
Fahimeh Fahimi Truski ${ }^{1}$ (id), Zohreh Ghotbeddin ${ }^{1,3^{*}}$ (D), Mohammad Reza Tabandeh ${ }^{2,3}$ (D), Mahdi Pourmahdi Borujeni ${ }^{4}$ (i)
}

1. Department of Physiology, Faculty of Veterinary Medicine, Shahid Chamran University of Ahvaz, Ahvaz, Iran.

2. Department of Biochemistry and Molecular Biology, Faculty of Veterinary Medicine, Shahid Chamran University of Ahvaz, Ahvaz, Iran

3. Stem Cell and Transgenic Technology Research Center, Shahid Chamran University of Ahvaz, Ahvaz, Iran.

4. Department of Food Hygiene, Faculty of Veterinary Medicine, Shahid Chamran University of Ahvaz, Ahvaz, Iran

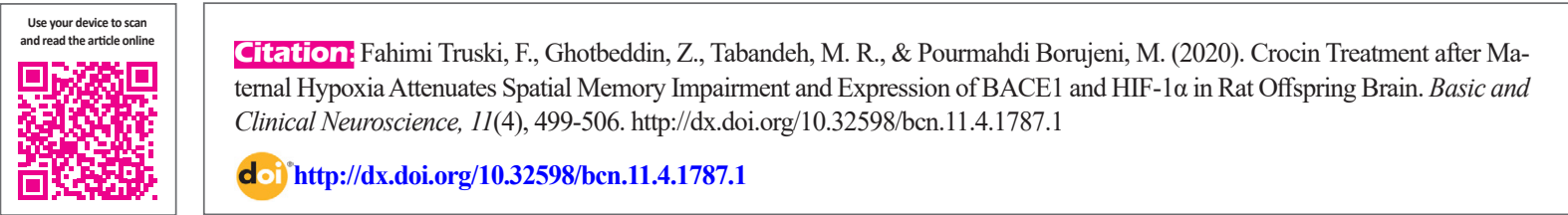

Article info:

Received: 22 Apr 2019

First Revision: 10 May 2019

Accepted: 11 Apr 2020

Available Online: $01 \mathrm{Jul} 2020$

Keywords:

Maternal hypoxia, Spatial memory, Rat offspring, Crocin, HIF1, BACE1

\begin{abstract}
AB S T RA C T
Introduction: Hypoxia via expression of Hypoxia-Inducible Factor-1 (HIF-1) is an important and effective factor in the onset and progression of memory disorders, such as Alzheimer Disease (AD). The activity of $\beta$-secretase (BACE1) is increased in hypoxia conditions. BACE1 triggers a cascade of pathological events resulting in AD. Crocin acts as a memoryimproving agent but its molecular mechanism is not well-known. Therefore, in this study, the effect of crocin on spatial memory, HIF-1 $\alpha$, and BACE1 gene expression was investigated in rat offspring under maternal hypoxia.

Methods: Female pregnant rats on the 20th day of pregnancy were divided into 4 groups, including sham, crocin-treated, hypoxia, and hypoxia group treated with crocin. In the hypoxia groups, pregnant rats were exposed to 7\% oxygen and 93\% nitrogen intensity for $3 \mathrm{~h}$. In the crocin-treated group, crocin (30 mg/kg) was injected at P14-28 (i.p). At the end, Morris water maze was used to assess spatial memory and real-time polymerase chain reaction was performed to measure the expression of BACE1 and HIF-1 $\alpha$ genes in the brain of offspring.

Results: Maternal hypoxia impaired memory compared with the sham group. However, crocin treatment improved cognitive behavior. HIF-1 $\alpha$ and BACE1 expressions were upregulated in the brain of offspring in the hypoxia group. Crocin treatment could attenuate the expression of both genes.

Conclusion: According to our results, down-regulation of HIF-1 $\alpha$ and BACE1 gene expressions in the brain of rat offspring after crocin treatment can be suggested as a molecular mechanism for crocin to improve spatial memory.
\end{abstract}




\section{Highlights}

- Alzheimer Disease (AD) is the most common form of dementia.

- Neural cell death in AD causes memory loss and cognitive impairment.

- Prenatal hypoxia is the most common form of hypoxia.

- The application of herbal compounds can be a suitable approach for reducing neurodegenerative complications.

\section{Plain Language Summary}

$\mathrm{AD}$ is the most common form of dementia, in which neural cell death causes memory loss and cognitive impairment. Hypoxia condition increases the incidence of AD. Prenatal hypoxia is the most common form of hypoxia, which causes abnormal brain development in the fetus and leads to learning and memory disability and its exposure to hypoxia as a critical period for memory development is contributed to attention loss and learning deficit. The hypoxia condition increases the expression and the enzymatic activity of BACE1, thus, the application of herbal compounds which can be suitable for reducing neurodegenerative complications. Also, crocin administration significantly inhibited apoptosis by decreasing the Bax-Bcl-2 ratio, which is due to its antioxidant activity.

\section{Introduction}

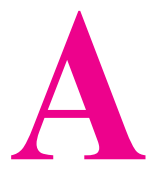

lzheimer Disease (AD) is the most common form of dementia, in which neural cell death causes memory loss and cognitive impairment. Pathological markers of $\mathrm{AD}$ are $\beta$-amyloid plaques formation outside the neurons, and hyperphosphorylated tau protein inside the neuron, especially in the areas contributed to learning and memory, such as the hippocampus (Ballatore, Lee, \& Trojanowski, 2007).

Hypoxia condition considerably increases the incidence of AD (Zhang et al., 2007). Because neurons are so sensitive to oxygen deficit, oxygen delivery impairment to the neuronal cells can lead to cell damage, and the risk of neurodegenerative diseases, such as AD and Parkinson disease is increased (Ogunshola \& Antoniou, 2009). Prenatal hypoxia is the most common form of hypoxia, which causes abnormal brain development in the fetus and leads to learning and memory disability in adolescent offspring rats (Wei et al., 2016).

As previous studies reported, prenatal exposure to hypoxia as a critical period for memory development is contributed to attention loss and learning deficit (Froehlich-Santino et al., 2016; Miguel et al., 2015).

The main molecular mechanism to induce neural dysfunction by hypoxia is unclear. $\beta$-amyloid Precursor Protein (APP) converts to the $\beta$-amyloid peptide $(\beta A)$ by consecutive proteolytic division from BACE1 and Presenilin-1 (PS1) $/ \gamma$-secretase (Zhou et al., 2016).

The hypoxia condition increases the expression and the enzymatic activity of BACE1 by up-regulating the level of BACE1 mRNA. Overexpression of BACE1 is mediated by HIF-1 $\alpha$ (Vetrivel, Zhang, Xu, \& Thinakaran, 2006). HIF- $1 \alpha$ is a major transcription factor responding to cellular oxygen deficit (Maxwell, \& Salnikow, 2004).

The application of herbal compounds can be a suitable approach for reducing neurodegenerative complications. Crocin, as a water-soluble carotenoid, presents in saffron stigma and has beneficial effects on learning, and memory and protects brain cells (Finley \& Gao, 2007). Several studies have shown that saffron extracts and crocin improved cognition. For example, ethanol induced memory impairment by inhibiting Long-Term Potentiation (LTP) and synaptic plasticity in the passive avoidance test performed by shuttle box test (Akhondzadeh \& Abbasi, 2006) and the oral administration of Cucumis sativus extract antagonized this impairment. Moreover, a single intraperitoneal injection of crocin $(30-60 \mathrm{mg} / \mathrm{kg}$ ) reversed memory disability induced by scopolamine in the passive avoidance task (Ghadami \& Pourmotabbed, 2009). Crocin administration significantly inhibited apoptosis by decreasing the Bax-Bcl-2 ratio, which is due to its antioxidant activity (Asadi et al., 2015).

Because the molecular mechanism of crocin for memory enhancing is not well known and hypoxia increases 
BACE1 activity and beta-amyloid formation from $b$ APP via HIF-1 $\alpha$ expression, the aim of this study was to investigate the effect of crocin on spatial memory and HIF- $1 \alpha$ and BACE1 genes expression in the brain of rat offspring affected by hypoxia during pregnancy.

\section{Materials and Methods}

\subsection{Animal experimental design}

Forty pregnant female rats were randomly divided into four groups (10 rats per group) as follows: A. Hypoxia group: to create hypoxia condition, rats were kept in hypoxia box and the concentration of oxygen in this chamber was decreased to $7 \%$ and the level of nitrogen was maintained at $93 \%$ for $3 \mathrm{~h}$ (Nalivaevaa et al., 2004); B. Sham operation (normal control group): female rats in this group were kept in the same room for the same time under normal oxygen content. In the hypoxia and sham-operated groups, all offspring were injected at P14 with saline for 2 weeks; C. Crocin-treated groups: female rats on the 20th day of gestation were maintained in a similar chamber for the same time under normal oxygen concentration.

At P14, all newborn animals were treated with crocin (30 mg/kg) (Bandegi, Rashidy-Pour, Vafaei, \& Ghadrdoost, 2014); D. Hypoxia group treated with crocin: first, these animals received hypoxia condition, and then all offspring were treated with crocin $(30 \mathrm{mg} / \mathrm{kg})$. All rats on the 20th day of gestation were individually placed in cages under standard laboratory conditions (ambient temperature of $23-25^{\circ} \mathrm{C}, 12 \mathrm{~h}$ light/dark alternate cycle with lights on between 7:00 AM and 7:00 PM, and food and water ad libitum). All experiments were carried out via a protocol approved by the Shahid Chamran University Animal Ethics Committee and were in complete compliance with the National Institutes of Health Guide for the Care and Use of Laboratory Animals. At the end of the treatment period, the spatial memory of all animals was checked using the Morris Water Maze (MWM) test. Next, animals were euthanized with a combination of ketamine $(100 \mathrm{mg} / \mathrm{kg})$ and xylazine $(10 \mathrm{mg} / \mathrm{kg})$. The brain was collected and kept at $-70^{\circ} \mathrm{C}$ until use for realtime polymerase chain reaction (PCR) analysis.

\subsection{Morris water maze task}

The experimental apparatus consisted of a circular water tank (160 cm wide and $60 \mathrm{~cm}$ high) surrounded by extra-maze cues. A submerged platform $(10 \mathrm{~cm}$ wide or $35 \mathrm{~cm}$ high) was placed $1.5 \mathrm{~cm}$ below the surface of the water. To assess for sensory, motor, or motivational skill, animals in each group were first trained by a task with a visible escape platform. The water temperature was 21 $23^{\circ} \mathrm{C}$. Data collection was automated by a video image motion analyzer. In a single training protocol, each rat completed three blocks (B1, B2, and B3) (each block consisted of four successive trials with four different releasing points) separated by a 30 min resting period. All experimental groups were tested with the lights on between 8:00 and 12:00 h. In each trial, rats were randomly released into the water in one of the four quadrants with their face toward the wall of the maze. During acquisition, the location of the platform remained constant and rats were allowed to swim for a duration of $60 \mathrm{~s}$ to find the hidden platform. After the animal found the platform, it was allowed to remain there for 30-35 s and then moved to the animal cage to wait for $30-35 \mathrm{~s}$ before the next trial. The time and distance needed to find the hidden platform was collected and analyzed later. A single probe trial was given $2 \mathrm{~h}$ after the last training trial to test the spatial memory in the MWM. In this trial, the platform was removed and the rat was allowed to swim for $60 \mathrm{~s}$. The time and distance spent in the target quadrant were analyzed as a measure of spatial memory retention (Frick, Stillner, \& Berger-Sweeney, 2000).

\subsection{Analysis of BACE1 and HIF1a expression}

Total RNA was isolated from the brain using the RNXTM isolation kit according to the manufacturer's procedure (Sinaclone, Iran) using $100 \mathrm{mg}$ of tissue. The concentration of extracted RNA was calculated at a wavelength of $260 \mathrm{~nm}$ using nanodrop spectrophotometry (Eppendorf, Germany). To detect the purity of RNA, its Optical Density (OD) absorption ratio at 260/280 nm was determined and the samples with a ratio of more than 1.8 were used for cDNA synthesis. Total RNA was converted to complementary DNA by reverse-transcription using random hexamer primers and YTA cDNA synthesis kit (Yektatajhiz, Iran).

For qRT-PCR, three replicates per sample were amplified and analyzed using a Roche Light-Cycler (Basel, Switzerland). Reactions were carried out in a 12.5 $\mu \mathrm{l}$ volume containing $6.25 \mu \mathrm{l}$ qPCRTM Green Master Kit for SYBR Green I® (Yektatajhiz, Iran), $0.25 \mu$ of each primer (200 nM), $3 \mu \mathrm{l}$ cDNA (100 ng), and 2.25 $\mu l$ nuclease-free water. Specific sets of primers (Bioneer, South Korea) designed for this study were HIF1 $\alpha$ (GenBank: NM_024359): 5'GTACCCTAA CTAGCCGAGGAAGAA-3' and 5'- GTGAATGTGGCCTGTGCAGT-3', GAPDH (GenBank: NM NM-001034055): 5'-CTCATCTACCTCTCCATCGTCTG-3' and 5'CCTGCTCTTGTCT GCCGGTGCTTG-3' and (GenBank: NM_019204): 5'-GCTGCAGTCAAGTCCATCAA-3' 
and 5'-ATTGCTGAGGAAGGATGGTG -3'. Reactions were performed in a $12.5 \mu \mathrm{l}$ mixture containing $6.25 \mu \mathrm{l}$ qPCRTM Green Master Kit for SYBR Green I $($ Yektatajhiz, Iran), $0.25 \mu \mathrm{l}$ of each primer (200 nM), $3 \mu \mathrm{l}$ cDNA (100 ng), and $2.25 \mu \mathrm{l}$ nuclease-free water. The PCR protocol used consisted of a 5 min denaturation at $94^{\circ} \mathrm{C}$ followed by 45 cycles of $94^{\circ} \mathrm{C}$ for $15 \mathrm{~s}$ and $60^{\circ} \mathrm{C}$ for $30 \mathrm{~s}$. Two separate reactions without cDNA or with RNA were performed in parallel as controls. The relative gene expression levels were determined using the comparative threshold cycle $(2-\Delta \Delta \mathrm{CT})$ method and Lightcycler 96® software. Validation of the assay to check whether the primer for the BACE1 and HIF1 $\alpha$ had similar amplification efficiencies was performed (Tabandeh, Jafari, Hosseini, \& Hashemitabar, 2015). All qPCR analyses were performed according to the Minimum Information for Publication of Quantitative Real-Time PCR Experiments (MIQE) guideline (Bustin et al., 2009).

\subsection{Data analysis}

All data were presented as Mean \pm SEM. Statistical analyses were conducted using SPSS V. 21 with a significance level of $\mathrm{P}<0.05$ and diagrams were plotted using the Microsoft Excel software. A One-Way Analysis of Variance (ANOVA) followed by Tukey's post-hoc test were used for comparing the obtained means in each parameter.

\subsection{Spatial memory assay using MWM}

As mentioned before, in MWM task has two stages; learning and probe to measure spatial memory. Traveled distance and the latency to find the hidden platform were measured in the learning stage, whereas the percentage of traveled distance and time spent in the target quarter were assessed in the probe stage.

\subsection{Traveled distance and latency time to find the hidden platform}

A comparison of the differences between groups in each block showed that crocin significantly decreased the traveled distance to find the hidden platform in block 3 compared with the hypoxia group $(\mathrm{P}<0.05)$. Traveled distance in the hypoxia group for block 3 was significantly higher than the sham and crocin groups $(\mathrm{P}<0.05)$ (Table 1).

The latency time to find the hidden platform for block 3 significantly decreased in the crocin and sham groups compared with the hypoxia group $(\mathrm{P}<0.05)$ (Table 1$)$.

\subsection{Traveled distance and time spent in the target quarter}

\section{Results}

Table 1. A comparison of the differences between groups in each block

\begin{tabular}{lccccc}
\hline Variabels & & Sham & Crocin & Hypoxia & Hypoxia+ Crocin \\
\hline & Block 1 & 9.89 & 9.4 & 12.14 & 13.06 \\
& Block 2 & 5.42 & 4 & 9.2 & 5.85 \\
Traveled distance $(\mathrm{m})$ & Block 3 & 4.11 & 2.27 & 8.7 & 2.89 \\
& SEMB1 & 1.2 & 0.88 & 1.01 & 1.12 \\
& SEMB2 & 2.01 & 0.78 & 0.49 & 1.33 \\
& SEMB3 & 0.98 & 0.35 & 1.31 & 41.32 \\
& Block 1 & 38.4 & 36.02 & 45.03 & 27.58 \\
& Block 2 & 22.27 & 16.94 & 33.25 & 23.28 \\
Block 3 & 17.78 & 11.77 & 33.78 & 2.87 \\
\hline SEMB1 & 3.25 & 2.56 & 4.09 & 4.72 \\
\hline SEMB2 & 6.9 & 2.61 & 2.34 & 5.58 \\
\hline
\end{tabular}


Table 2. A comparison of the Mean \pm SEM between Target quadrant time (s) and Target traveled distance $(\mathrm{m})$

\begin{tabular}{lcc}
\hline \multicolumn{2}{c}{ Variabels } & Mean \pm SEM \\
\hline & Sham & $44.58 \pm 4.12$ \\
Target quadrent time $(\mathrm{s})$ & Crocin & $49.81 \pm 6.14$ \\
& Hypoxia & $24.79 \pm 2.14$ \\
& Hypoxia+ Crocin & $41.78 \pm 3.72$ \\
& Sham & $42.01 \pm 3.55$ \\
Target traveled distance $(\mathrm{m})$ & Crocin & $47.23 \pm 5.16$ \\
& Hypoxia & $25.24 \pm 1.39$ \\
& Hypoxia+ Crocin & $38.92 \pm 3.13$ \\
\hline
\end{tabular}

NEUR SCIENCE

Table 3. Comparing the mRNA level of HIF-1a in the brain tissues

\begin{tabular}{cccc}
\hline \multicolumn{2}{c}{ Variabels } & & \multicolumn{2}{c}{ Mean \pm SEM } \\
\hline & Sham & 1.0837 & 0.03917 \\
HIF1- $\alpha$ & Crocin & 0.8297 & 0.02209 \\
& Hypoxia & 3.3827 & 0.16225 \\
& Hypoxia+ Crocin & 1.7122 & 0.04555 \\
BACE1 & Sham & 1.1001 & 0.04737 \\
& Crocin & 0.9313 & 0.04485 \\
& Hypoxia & 3.7508 & 0.16709 \\
& Hypoxia+ Crocin & 2.1205 & 0.11257 \\
\hline
\end{tabular}

NEUR:SCIENCE

Traveled distance in the target quarter significantly decreased $(\mathrm{P}<0.05)$ in the hypoxia group than in other groups (Table 2).

Analysis of the time spent in the target quarter showed a significant decrease in the hypoxia group compared with other groups $(\mathrm{P}<0.05)$ (Table 2$)$.

\subsection{HIF-1a gene expression}

Comparing the mRNA level of HIF-1 $\alpha$ in the brain tissues of 30-day-old offspring indicated a significant difference between groups. HIF-1 $\alpha$ gene expression in the hypoxia group significantly increased in comparison with the sham and crocin groups $(\mathrm{P}<0.001)$ and hypoxia group treated with crocin $(\mathrm{P}<0.01)$. However, crocin treatment significantly decreased HIF- $1 \alpha$ gene expression (Table 3A).

\subsection{BACE1 gene expression}

The mean of BACE1 gene expression in the brain tissues of offspring in the hypoxia group showed a significant increase compared with the sham and crocin groups $(\mathrm{P}<0.001)$. The mRNA level of BACE1 significantly decreased in hypoxic offspring after treatment with crocin $(\mathrm{P}<0.01)$ (Table 3).

\section{Discussion}

Hypoxia is one of the most common types of stress during pregnancy that can affect the behavior of the infants until the end of their lives by altering the function of the 
hippocampus and disrupting neural growth and development. Crocin is considered a proper treatment option to prevent the neural damages caused by hypoxia. Thus, the present study was conducted to investigate the effects of crocin on spatial memory and the expression of genes associated with the formation of $\beta$-amyloid in the brains of rat offspring affected by hypoxia during pregnancy. Based on our results, spatial learning in the hypoxia group showed a significant decrease compared with the sham group. In this regard, several previous studies suggested that one of the most significant complications of hypoxia is long-term memory impairment. Pregnancy hypoxia not only disrupts navigation but also affects memory retrieval in adolescence. A decrease is observed in the learning and memory capabilities of adolescents whose mothers suffered from hypoxia during pregnancy (Wei, et al., 2016).

Another study was conducted to investigate the pathological effects of hypoxia during pregnancy in transgenic mice (APPSwe/PS1A246E), and results indicated a significant decrease in learning, spatial memory, and synaptogenesis in their offspring. In addition, a high level of APP, a low level of neprilysin (an amyloid beta-degrading enzyme), and an increase in the accumulation of $\beta$-amyloid were observed in the brains of mice that suffered from oxygen deficiency during pregnancy (Zhang et al., 2013).

The results of the MWM task also showed that an increase in traveled distance and time to find the hidden platform, as well as a higher level of learning and spatial memory impairment in the offspring born to mothers with hypoxia (Zhang, \& Le, 2010). Hypoxia plays an important role in the pathogenesis of AD by increasing the accumulation of $\beta$-amyloid, increasing tau protein phosphorylation, disrupting the function of the bloodbrain barrier, and degenerating neurons (Zhang, \& Le, 2010). The $\beta$-amyloid hypothesis is considered as the main hypothesis for cell apoptosis and ultimately progression of AD. Through their proteolytic function, BACE1 and PS1 $/ \gamma$-secretase enzymes convert the betasite APP to $\beta$-amyloid (Suganthy, Malar, \& Devi, 2016).

To understand the molecular mechanism of memory impairment in the hypoxia group, HIF- $1 \alpha$ and BACE1 gene expression levels were measured, and the results indicated a significant increase in the expression of these genes in the hypoxia group compared with the sham and crocin groups. In this regard, other researchers suggested that the activity of the BACE1 enzyme is increased by the up-regulation of the mRNA1 of the enzyme. Increased activity of the enzyme following hypoxia leads to the increased production of $\beta$-amyloid from the beta-site APP. By expressing HIF-1 $\alpha$, hypoxia causes an increase in the activity of the BACE1 enzyme, which is a direct relationship between HIF-1 $\alpha$ expression and BACE1 enzyme activity (Zhang er al, 2007).

The continuous divisions of $\gamma$-secretase and $\beta$-secretase (BACE1) enzymes in the beta-site APP lead to the production of $\beta$-amyloid with two types of $\mathrm{C}$-terminals ( $\beta$ A42 and $\beta A 40)$. The accumulation of $\beta A 40$ in the brain tissue is the main cause of brain damage in $\mathrm{AD}$ (Tamagno, Guglielmotto, Monteleone, \& Tabaton, 2012). Several studies have shown that protein levels and BACE1 activity are elevated in brain regions, which are affected by $\mathrm{AD}$, suggesting that the abnormal activity of BACE1 plays an important role in the pathogenesis of AD (Tamagno et al., 2012; Yang et al., 2003). Wen et al. showed a significant increase in the activity and expression of BACE1 in rats affected by transient cerebral ischemia (Wen, Onyewuchi, Yang, Liu, \& Simpkins, 2004).

The use of effective herbal compounds to prevent or reduce the complications of neurodegenerative diseases has been considered in recent years. Although the antioxidant effect of these compounds has been proven, the protective molecular mechanism of them is still unknown. Crocin is one of these compounds, which have been clinically considered in the treatment of $\mathrm{AD}$. The results of the present study indicated an improvement in the spatial memory of the crocin-treated group compared with the hypoxia group. Previous studies suggested that saffron extract contains active ingredients, such as crocin that plays a significant role in learning, memory improvement, and oxygen diffusion in different tissues (Naghizadeh, Mansouri, \& Mashhadian, 2010).

Naghizadeh et al. investigated the effects of crocin (100 $\mathrm{mg} / \mathrm{kg}$ ) on the cognitive functions in streptozotocin-injected rats. Streptozotocin injection caused memory impairment and crocin treatment improved cognitive functions (Naghizadeh, Mansouri, Ghorbanzadeh, Farbood, \& Sarkaki, 2013). Hosseinzadeh et al. investigated the effect of crocin and saffron extract on cognition and memory impairment caused by ischemia through MWM and suggested that i.p. injection of ischemic rats with crocin (5-25 $\mathrm{mg} / \mathrm{kg}$ ) and saffron (50-250 mg/kg) extract caused an improvement in cognition and memory (Hosseinzadeh, Sadeghnia, Ghaeni, Motamedshariaty, \& Mohajeri, 2012). The antioxidant effect of saffron and crocin can be considered as a potential protection mechanism of the central nervous system against the oxidative damage caused by hypoxia. Other findings also showed that saffron extract can reduce the oxidative stress caused by chronic stress in the hippocampus (Ghadrdoost et al., 2011). 
As mentioned before, the protective molecular mechanism of crocin is still unknown, and no studies have been conducted in this regard. In the present study, HIF-1 $\alpha$ and BACE1 expression leves were measured to understand the protective molecular mechanism of crocin after hypoxiainduced memory impairment, and the results showed a significant decrease in the expression of the genes in the treated group with crocin compared with the hypoxia group.

Based on the results of the present study, maternal hypoxia increased the transcription of BACE1 enzyme mRNA1 in offspring brains by increasing the expression of the HIF-1 $\alpha$ gene. Subsequently, the conversion of the beta-site APP to $\beta$-Amyloid was facilitated, which results in memory impairment. Crocin treatment improved memory impairment by decreasing the expression of HIF- $1 \alpha$ and BACE1 genes.

\section{Ethical Considerations}

\section{Compliance with ethical guidelines}

All ethical principles were considered in this article.

\section{Funding}

This research was supported by the Research Council of the Shahid Chamran University of Ahvaz.

\section{Authors' contributions}

All authors contributed equally in preparing all parts of the research.

\section{Conflict of interest}

The authors declare that they have no conflict of interest.

\section{Acknowledgments}

We are grateful to the Research Council of the Shahid Chamran University of Ahvaz for financial support.

\section{Reference}

Ballatore, C., Lee, V. M. Y., \& Trojanowski, J. Q. (2007). Tau-mediated neurodegeneration in Alzheimer's disease and related disorders. Nature Reviews Neuroscience, 8(9), 663-72.[DOI:10.1038/nrn2194] [PMID]

Zhang, X., Zhou, K., Wang, R., Cui, J., Lipton, S. A., \& Liao, F. F (2007). Hypoxia-inducible factor 1a (HIF-1a)-mediated hypoxia increases BACE1 expression and $\beta$-amyloid generation. Jour- nal of Biological Chemistry, 282(15), 10873-80. [DOI:10.1074/jbc M608856200] [PMID]

Ogunshola, O. O., \& Antoniou, X. (2009). Contribution of hypoxia to Alzheimer's disease: Is HIF-1 a a mediator of neurodegeneration? Cellular and Molecular Life Sciences, 66(22), 3555-63. [DOI:10.1007/ s00018-009-0141-0] [PMID]

Wei, B., Li, L., He, A., Zhang, Y., Sun, M., \& Xu, Z. (2016). Hippocampal NMDAR-Wnt-Catenin signaling disrupted with cognitive deficits in adolescent offspring exposed to prenatal hypoxia Brain Research, 1631, 157-64. [DOI:10.1016/j.brainres.2015.11.041] [PMID]

Froehlich-Santino, W., Tobon, A. L., Cleveland, S., Torres, A., Phillips, J.,\& Cohen, B., et al. (2014). Prenatal and perinatal risk factors in a twin study of autism spectrum disorders. Journal of Psychiatric Research, 54, 100-8. [DOI:10.1016/j.jpsychires.2014.03.019] [PMID] [PMCID]

Miguel, P. M., Schuch, C. P., Rojas, J. J., Carletti, J. V., Deckmann, I., \& Martinato, L. H. M., et al. (2015). Neonatal hypoxia-ischemia induces attention-deficit hyperactivity disorder-like behavior in rats. Behavioral Neuroscience, 129(3), 309-20. [DOI:10.1037/ bne0000063] [PMID]

Zhou, D., Liu, H., Li, C., Wang, F., Shi, Y., \& Liu, L., et al. (2016). Atorvastatin ameliorates cognitive impairment, $\mathrm{A} \beta 1-42$ production and Tau hyperphosphorylation in APP/PS1 transgenic mice. Metabolic Brain Disease, 31(3), 693-703.[DOI:10.1007/s11011-0169803-4] [PMID]

Vetrivel, K. S., Zhang, Y. W., Xu, H., \& Thinakaran, G. (2006). Pathological and physiological functions of presenilins. Molecular Neurodegeneration, 1(1), 4. [DOI:10.1186/1750-1326-1-4] [PMID] [PMO CID]

Maxwell, P., \& Salnikow, K. (2004). HIF-1, an oxygen and metal responsive transcription factor. Cancer biology \& therapy, 3(1), 29-35. [DOI:10.4161/cbt.3.1.547] [PMID]

Finley, J. W., \& Gao, S. (2017). A perspective on Crocus sativus L.(Saffron) constituent crocin: A potent water-soluble antioxidant and potential therapy for Alzheimer's disease. Journal of Agricultural and Food Chemistry, 65(5), 1005-20. [DOI:10.1021/acs. jafc.6b04398] [PMID]

Akhondzadeh, S., \& Abbasi, S. H. (2006). Herbal medicine in the treatment of Alzheimer's disease. American Journal of Alzheimer's Disease $\mathcal{E}$ Other Dementias ${ }^{\circledR}, 21(2), 113-8$. [DOI:10.1177/15333175 0602100211] [PMID]

Ghadami, M. R., Pourmotabbed, A. (2009). [The effect of crocin on scopolamine induced spatial learning and memory deficits in rats (Persian)]. Physiology and Pharmacology, 12(4), 287-95. http://ppi. phypha.ir/article-1-462-en.pdf

Asadi, F., Jamshidi, A. H., Khodagholi, F., Yans, A., Azimi, L., \& Faizi, M., et al. (2015). Reversal effects of crocin on amyloid $\beta$-induced memory deficit: Modification of autophagy or apoptosis markers. Pharmacology Biochemistry and Behavior, 139, 47-58. [DOI:10.1016/j. pbb.2015.10.011] [PMID]

Nalivaevaa, N. N., Fisk, L., Kochkina, E. G., Plesneva, S. A., Zhuravin, I. A., \&Babusikova, E. V. A., et al. (2004). Effect of hypoxia/ischemia and hypoxic preconditioning/reperfusion on expression of some amyloid-degrading enzymes. Annals of the New York Academy of Sciences, 1035(1), 21-33. [DOI:10.1196/annals.1332.002] [PMID] 
Bandegi, A. R., Rashidy-Pour, A., Vafaei, A. A., \& Ghadrdoost, B. (2014). Protective effects of Crocus sativus L. extract and crocin against chronic-stress induced oxidative damage of brain, liver and kidneys in rats. Advanced Pharmaceutical Bulletin, 4(Suppl 2), 493-99. [DOI:10.5681/apb.2014.073] [PMCID] [PMID]

Frick, K. M., Stillner, E. T., \& Berger-Sweeney, J. (2000). Mice are not little rats: Species differences in a one-day water maze task. Neuroreport, 11(16), 3461-5. [DOI:10.1097/00001756-200011090-00013] [PMID]

Tabandeh, M. R., Jafari, H., Hosseini, S. A., \& Hashemitabar, M. (2015). Ginsenoside Rb1 stimulates adiponectin signaling in C2C12 muscle cells through up-regulation of AdipoR1 and AdipoR2 proteins. Pharmaceutical Biology, 53(1), 125-32. [DOI:10.3109/ 13880209.2014.912237] [PMID]

Bustin, S. A., Benes, V., Garson, J. A., Hellemans, J., Huggett, J., \& Kubista, M., et al. (2009). The MIQE Guidelines: M inimum I nformation for Publication of $Q$ uantitative Real-Time PCR E xperiments. Clinical Chemistry, 55(4), 611-22. [DOI:10.1373/ clinchem.2008.112797] [PMID]

Zhang, X., Li, L., Zhang, X., Xie, W., Li, L., \& Yang, D., et al. (2013). Prenatal hypoxia may aggravate the cognitive impairment and Alzheimer's disease neuropathology in APPSwe/PS1A246E transgenic mice. Neurobiology of Aging, 34(3), 663-78. [DOI:10.1016/j. neurobiolaging.2012.06.012] [PMID]

Zhang, X., \& Le, W. (2010). Pathological role of hypoxia in Alzheimer's disease. Experimental Neurology, 223(2), 299-303. [DOI:10.1016/j. expneurol.2009.07.033] [PMID]

Suganthy, N., Malar, D. S., \& Devi, K. P. (2016). Rhizophora mucronata attenuates beta-amyloid induced cognitive dysfunction, oxidative stress and cholinergic deficit in Alzheimer's disease animal model. Metabolic Brain Disease, 31(4), 937-49. [DOI:10.1007/s11011016-9831-0] [PMID]

Tamagno, E., Guglielmotto, M., Monteleone, D., \& Tabaton, M. (2012). Amyloid- $\beta$ production: Major link between oxidative stress and BACE1. Neurotoxicity Research, 22(3), 208-19.[DOI:10.1007/ s12640-011-9283-6] [PMID]

Yang, L. B., Lindholm, K., Yan, R., Citron, M., Xia, W., \&Yang, X. L., et al. (2003). Elevated $\beta$-secretase expression and enzymatic activity detected in sporadic Alzheimer disease. Nature Medicine, 9(1), 3-4. [DOI:10.1038/nm0103-3] [PMID]

Wen, Y., Onyewuchi, O., Yang, S., Liu, R., \& Simpkins, J. W. (2004). Increased $\beta$-secretase activity and expression in rats following transient cerebral ischemia. Brain Research, 1009(1-2), 1-8. [DOI:10.1016/j.brainres.2003.09.086] [PMID]

Naghizadeh, B., Mansouri, S. M. T., \& Mashhadian, N. V. (2010). Crocin attenuates cisplatin-induced renal oxidative stress in rats. Food and Chemical Toxicology, 48(10), 2650-5. [DOI:10.1016/j. fct.2010.06.035] [PMID]

Naghizadeh, B., Mansouri, M. T., Ghorbanzadeh, B., Farbood, Y., \& Sarkaki, A. (2013). Protective effects of oral crocin against intracerebroventricular streptozotocin-induced spatial memory deficit and oxidative stress in rats. Phytomedicine, 20(6), 537-42. [DOI:10.1016/j.phymed.2012.12.019] [PMID]

Hosseinzadeh, H., Sadeghnia, H. R., Ghaeni, F. A., Motamedshariaty, V. S., \& Mohajeri, S. A. (2012). Effects of saffron (Crocus sativus L.) and its active constituent, crocin, on recognition and spatial memory after chronic cerebral hypoperfusion in rats. Phytotherapy Research, 26(3), 381-6. [DOI:10.1002/ptr.3566] [PMID]
Ghadrdoost, B., Vafaei, A. A., Rashidy-Pour, A., Hajisoltani, R, Bandegi, A. R., \& Motamedi, F., et al. (2011). Protective effects of saffron extract and its active constituent crocin against oxidative stress and spatial learning and memory deficits induced by chronic stress in rats. European Journal of Pharmacology, 667(1-3), 222-9. [DOI:10.1016/j.ejphar.2011.05.012] [PMID] 